\title{
Second Hankel determinant for a class of analytic functions of complex order defined by convolution
}

\begin{abstract}
In this paper, we obtain the Fekete-Szegö inequalities for the functions of complex order defined by convolution. Also, we find upper bounds for the second Hankel determinant $\left|a_{2} a_{4}-a_{3}^{2}\right|$ for functions belonging to the class $S_{\gamma}^{b}(g(z) ; A, B)$.
\end{abstract}

1. Introduction. Let $\mathcal{A}$ denote the class of analytic functions of the form:

$$
f(z)=z+\sum_{k=2}^{\infty} a_{k} z^{k} \quad(z \in \mathbb{U}=\{z: z \in \mathbb{C} \text { and }|z|<1\})
$$

and $\mathcal{S}$ be the subclass of $\mathcal{A}$ consisting of univalent functions. Furthermore, let $\mathcal{P}$ be a family of functions $p(z) \in \mathcal{A}$.

Let $g(z) \in \mathcal{S}$ be given by

$$
g(z)=z+\sum_{k=2}^{\infty} b_{k} z^{k}
$$

The Hadamard product (or convolution) of $f(z)$ and $g(z)$ is given by

$$
(f * g)(z)=z+\sum_{k=2}^{\infty} a_{k} b_{k} z^{m}=(g * f)(z) .
$$

2010 Mathematics Subject Classification. 30C45.

Key words and phrases. Fekete-Szegö inequality, second Hankel determinant, convolution, complex order. 
If $f$ and $g$ are analytic functions in $\mathbb{U}$, we say that $f$ is subordinate to $g$, written $f \prec g$ if there exists a Schwarz function $w$, which is analytic in $\mathbb{U}$ with $w(0)=0$ and $|w(z)|<1$ for all $z \in \mathbb{U}$, such that $f(z)=g(w(z))$. Furthermore, if the function $g$ is univalent in $\mathbb{U}$, then we have the following equivalence (see [6] and [19]):

$$
f(z) \prec g(z) \Leftrightarrow f(0)=g(0) \text { and } f(\mathbb{U}) \subset g(\mathbb{U}) .
$$

For complex parameters $\alpha_{1}, \ldots, \alpha_{q}$ and $\beta_{1}, \ldots, \beta_{s}\left(\beta_{j} \notin \mathbb{Z}_{0}^{-}=\{0,-1\right.$, $-2, \ldots\} ; j=1,2, \ldots, s)$, we now define the generalized hypergeometric function ${ }_{q} F_{s}\left(\alpha_{1}, \ldots, \alpha_{q} ; \beta_{1}, \ldots, \beta_{s} ; z\right)$ by (see, for example, [29, p. 19])

$$
{ }_{q} F_{s}\left(\alpha_{1}, \ldots, \alpha_{q} ; \beta_{1}, \ldots, \beta_{s} ; z\right)=\sum_{k=0}^{\infty} \frac{\left(\alpha_{1}\right)_{k} \ldots\left(\alpha_{q}\right)_{k}}{\left(\beta_{1}\right)_{k} \ldots\left(\beta_{s}\right)_{k}} \cdot \frac{z^{k}}{k !}
$$

$\left(q \leq s+1 ; q, s \in \mathbb{N}_{0}=\mathbb{N} \cup\{0\} ; \mathbb{N}=\{1,2, \ldots\} ; z \in \mathbb{U}\right)$, where $(\theta)_{\nu}$ is the Pochhammer symbol defined, in terms of the Gamma function $\Gamma$, by

$$
\begin{aligned}
(\theta)_{\nu} & =\frac{\Gamma(\theta+\nu)}{\Gamma(\theta)} \\
& = \begin{cases}1 & \left(\nu=0 ; \theta \in \mathbb{C}^{*}=\mathbb{C} \backslash\{0\}\right), \\
\theta(\theta+1) \ldots(\theta+\nu-1) & (\nu \in \mathbb{N} ; \theta \in \mathbb{C}) .\end{cases}
\end{aligned}
$$

It corresponds to the function $h_{q, s}\left(\alpha_{1}, \beta_{1} ; z\right)=h\left(\alpha_{1}, \ldots \alpha_{q} ; \beta_{1} \ldots, \beta_{s} ; z\right)$, defined by

$$
\begin{aligned}
h_{q, s}\left(\alpha_{1}, \beta_{1} ; z\right) & =z_{q} F_{s}\left(\alpha_{1}, \ldots, \alpha_{q} ; \beta_{1}, \ldots, \beta_{s} ; z\right) \\
& =z+\sum_{k=2}^{\infty} \Gamma_{k}\left(\alpha_{1}\right) z^{k}
\end{aligned}
$$

where

$$
\Gamma_{k}\left(\alpha_{1}\right)=\frac{\left(\alpha_{1}\right)_{k-1} \ldots\left(\alpha_{q}\right)_{k-1}}{\left(\beta_{1}\right)_{k-1} \ldots\left(\beta_{s}\right)_{k-1}(k-1) !} .
$$

In [13] El-Ashwah and Aouf defined the operator $I_{q, s, \lambda}^{m, \ell}\left(\alpha_{1}, \beta_{1}\right) f(z)$ as follows:

$$
\begin{aligned}
I_{q, s, \lambda}^{0, \ell}\left(\alpha_{1}, \beta_{1}\right) f(z)= & f(z) * h_{q, s}\left(\alpha_{1}, \beta_{1} ; z\right) \\
I_{q, s, \lambda}^{1, \ell}\left(\alpha_{1}, \beta_{1}\right) f(z)= & (1-\lambda)\left(f(z) * h_{q, s}\left(\alpha_{1}, \beta_{1} ; z\right)\right) \\
& +\frac{\lambda}{(1+\ell) z^{\ell-1}}\left[z^{\ell}\left(f(z) * h_{q, s}\left(\alpha_{1}, \beta_{1} ; z\right)\right)\right]^{\prime}
\end{aligned}
$$

and

$$
I_{q, s, \lambda}^{m, \ell}\left(\alpha_{1}, \beta_{1}\right) f(z)=I_{q, s, \lambda}^{1, \ell}\left(I_{q, s, \lambda}^{m-1, \ell}\left(\alpha_{1}, \beta_{1}\right) f(z)\right) .
$$


If $f \in A$, then from (1.1) and (1.7), we can easily see that

$$
I_{q, s, \lambda}^{m, \ell}\left(\alpha_{1}, \beta_{1}\right) f(z)=z+\sum_{k=2}^{\infty}\left[\frac{1+\ell+\lambda(k-1)}{1+\ell}\right]^{m} \Gamma_{k}\left(\alpha_{1}\right) a_{k} z^{k},
$$

where $m \in \mathbb{Z}=\{0, \pm 1, \ldots\}, \ell \geq 0$ and $\lambda \geq 0$.

We note that when $\ell=0$, the operator

$$
I_{q, s, \lambda}^{m, 0}\left(\alpha_{1}, \beta_{1}\right) f(z)=D_{\lambda}^{m}\left(\alpha_{1}, \beta_{1}\right) f(z)
$$

was studied by Selvaraj and Karthikeyan [28]. We also note that:

(i) $I_{q, s, \lambda}^{0, \ell} f(z)=H_{q, s}\left(\alpha_{1}, \beta_{1}\right) f(z)$ (see Dziok and Srivastava $\left.[11,12]\right)$;

(ii) For $q=s+1, \alpha_{i}=1(i=1, \ldots, s+1)$ and $\beta_{j}=1(j=1, \ldots, s)$, we get the operator $I(m, \lambda, \ell)$ (see Catas [7], Prajapat [24] and El-Ashwah and Aouf [14]);

(iii) For $q=s+1, \alpha_{i}=1(i=1, \ldots, s+1), \beta_{j}=1(j=1, \ldots, s), \lambda=1$ and $\ell=0$, we obtain the Sălăgean operator $D^{m}$ (see Sălăgean [27]);

(iv) For $q=s+1, \alpha_{i}=1(i=1, \ldots, s+1), \beta_{j}=1(j=1, \ldots, s)$ and $\lambda=1$, we get the operator $I_{\ell}^{m}$ (see Cho and Srivastava [8] and Cho and Kim [9]).

(v) For $q=s+1, \alpha_{i}=1(i=1, \ldots, s+1), \beta_{j}=1(j=1, \ldots, s)$ and $\ell=0$, we obtain the operator $D_{\lambda}^{m}$ (see Al-Oboudi [2]).

By specializing the parameters $m, \lambda, \ell, q, s, \alpha_{i}(i=1, \ldots, q)$ and $\beta_{j}$ $(j=1, \ldots, s)$ we obtain:

(i) $I_{2,1, \lambda}^{m, \ell}(n+1,1 ; 1) f(z)=I_{\lambda}^{m, \ell}(n) f(z)=z+\sum_{k=2}^{\infty}\left[\frac{1+\ell+\lambda(k-1)}{1+\ell}\right]^{m} \frac{(n+1)_{k-1}}{(1)_{k-1}} a_{k} z^{k}$ $(n>-1)$;

(ii) $I_{2,1, \lambda}^{m, \ell}(a, 1 ; c) f(z)=I_{\lambda}^{m, \ell}(a ; c) f(z)=z+\sum_{k=2}^{\infty}\left[\frac{1+\ell+\lambda(k-1)}{1+\ell}\right]^{m} \frac{(a)_{k-1}}{(c)_{k-1}} a_{k} z^{k}$ $\left(a \in \mathbb{R} ; c \in \mathbb{R} \backslash \mathbb{Z}_{0}^{-}\right) ;$

(iii) $I_{2,1, \lambda}^{m, \ell}(2,1 ; n+1) f(z)=I_{\lambda, n}^{m, \ell} f(z)=z+\sum_{k=2}^{\infty}\left[\frac{1+\ell+\lambda(k-1)}{1+\ell}\right]^{m} \frac{(2)_{k-1}}{(n+1)_{k-1}} a_{k} z^{k}$ $(n \in \mathbb{Z} ; n>-1)$.

In 1976, Noonan and Thomas [23] discussed the $q$ th Hankel determinant of a locally univalent analytic function $f(z)$ for $q \geq 1$ and $n \geq 1$ which is defined by

$$
H_{q}(n)=\left|\begin{array}{cccc}
a_{n} & a_{n+1} & \ldots & a_{n+q-1} \\
a_{n+1} & a_{n+2} & \ldots & a_{n+q} \\
\vdots & \vdots & \ldots & \vdots \\
a_{n+q-1} & a_{n+q} & \ldots & a_{n+2 q-2}
\end{array}\right| .
$$

For our present discussion, we consider the Hankel determinant in the case $q=2$ and $n=2$, i.e. $H_{2}(2)=a_{2} a_{4}-a_{3}^{2}$. This is popularly known as the second Hankel determinant of $f$.

In this paper, we define the following class $S_{\gamma}^{b}(g(z) ; A, B)(0 \leq \gamma \leq 1, b \in$ $\left.\mathbb{C}^{*}=\mathbb{C} \backslash\{0\}\right)$ as follows: 
Definition 1. Let $0 \leq \gamma \leq 1, b \in \mathbb{C}^{*}$. A function $f(z) \in \mathcal{A}$ is said to be in the class $S_{\gamma}^{b}(g(z) ; A, B)$ if

$$
1+\frac{1}{b}\left((1-\gamma) \frac{(f * g)(z)}{z}+\gamma(f * g)^{\prime}(z)-1\right) \prec \frac{1+A z}{1+B z}
$$

$\left(b \in \mathbb{C}^{*} ; 0 \leq \gamma \leq 1 ;-1 \leq B<A \leq 1 ; z \in \mathbb{U}\right)$, which is equivalent to say that

$$
\left|\frac{(1-\gamma) \frac{(f * g)(z)}{z}+\gamma(f * g)^{\prime}(z)-1}{b(A-B)-B\left[(1-\gamma) \frac{(f * g)(z)}{z}+\gamma(f * g)^{\prime}(z)-1\right]}\right|<1 .
$$

We note that for suitable choices of $b, \gamma$ and $g(z)$ we obtain the following subclasses:

(i) $S_{\gamma}^{b}\left(\frac{z}{1-z} ; A, B\right)=S_{\gamma}^{b}(A, B)\left(0 \leq \gamma \leq 1, b \in \mathbb{C}^{*},-1 \leq B<A \leq 1\right)$ (see Bansal [5]);

(ii) $S_{0}^{(1-\rho) e^{-i \theta} \cos \theta}\left(z+\sum_{k=2}^{\infty} \frac{(\alpha)_{k-1}}{(\beta)_{k-1}} z^{k} ; 1,-1\right)=\mathcal{R}_{\alpha, \beta}(\theta, \rho)\left(\frac{-\pi}{2}<\theta<\frac{\pi}{2}\right.$, $0 \leq \rho<1, \alpha \in \mathbb{C}, \beta \in \mathbb{C} \backslash \mathbb{Z}_{0}^{-}$) (see Mishra and Kund [21]);

(iii) $S_{0}^{(1-\rho) e^{-i \alpha} \cos \alpha}\left(z+\sum_{k=2}^{\infty} \frac{(\lambda+1)_{k-1}}{(m)_{k-1}} k^{n} z^{k} ; 1,-1\right)=S_{m}^{\lambda, n}(\alpha, \sigma) \quad(m \in \mathbb{N}$; $n, \lambda \in \mathbb{N}_{0} ;|\alpha|<\frac{\pi}{2} ; 0 \leq \sigma<1$ ) (see Mohammed and Darus [22]);

(iv) $S_{1}^{1}\left(z+\sum_{k=2}^{\infty}[1+(\alpha \mu k+\alpha-\mu)(k-1)]^{\sigma}(\rho)_{k-1} z^{k} ; 1,-1\right)=R_{\alpha, \mu}(\sigma, \rho)$ $\left(0 \leq \mu \leq \alpha \leq 1 ; \rho, \sigma \in \mathbb{N}_{0}\right)$ (see Abubaker and Darus [1]);

(v) $S_{\gamma}^{b}\left(z+\sum_{k=2}^{\infty} k^{m} z^{k} ; A, B\right)=G_{m}(\gamma, b)\left(b \in \mathbb{C}^{*}, 0 \leq \gamma \leq 1, m \in \mathbb{N}_{0}\right)$ (see Aouf [3]).

Also, we note that:

(i) $S_{\gamma}^{b}\left(z+\sum_{k=2}^{\infty}\left[\frac{1+\ell+\lambda(k-1)}{1+\ell}\right]^{m} \Gamma_{k}\left(\alpha_{1}\right) z^{k} ; A, B\right)=S_{\gamma}^{b}\left(\lambda, \ell, m, q, s, \alpha_{1}, \beta_{1} ; A, B\right)$

$$
\begin{gathered}
=\left\{f(z) \in \mathcal{A}: 1+\frac{1}{b}\left((1-\gamma) \frac{I_{q, s, \lambda}^{m, \ell}\left(\alpha_{1}, \beta_{1}\right) f(z)}{z}+\gamma\left(I_{q, s, \lambda}^{m, \ell}\left(\alpha_{1}, \beta_{1}\right) f(z)\right)^{\prime}-1\right)\right. \\
\prec \frac{1+A z}{1+B z}, \quad\left(b \in \mathbb{C}^{*} ; 0 \leq \gamma \leq 1 ; m \in \mathbb{N}_{0} ; \ell \geq 0 ; \lambda \geq 0 ; q \leq s+1 ;\right. \\
\left.\left.q, s \in \mathbb{N}_{0} ; \quad z \in \mathbb{U}\right)\right\} ;
\end{gathered}
$$

(ii) $S_{\gamma}^{b}\left(z+\sum_{k=2}^{\infty}\left[\frac{1+\ell}{1+\ell+\lambda(k-1)}\right]^{m} z^{k} ; A, B\right)=S_{\gamma}^{b}(\lambda, \ell, m ; A, B)$

$$
\begin{aligned}
& =\left\{f(z) \in \mathcal{A}: 1+\frac{1}{b}\left((1-\gamma) \frac{J^{m}(\lambda, \ell) f(z)}{z}+\gamma\left(J^{m}(\lambda, \ell) f(z)\right)^{\prime}-1\right)\right. \\
& \left.\prec \frac{1+A z}{1+B z},\left(b \in \mathbb{C}^{*} ; 0 \leq \gamma \leq 1 ; m \in \mathbb{N}_{0} ; \ell \geq 0 ; \lambda \geq 0 ; \quad z \in \mathbb{U}\right)\right\} ;
\end{aligned}
$$


(iii) $S_{\gamma}^{(1-\rho) \cos \eta e^{-i \eta}}(g(z) ; A, B)=S^{\gamma}[\rho, \eta, A, B, g(z)]$

$$
\begin{aligned}
=\{f(z) & \in \mathcal{A}: e^{i \eta}\left[(1-\gamma) \frac{(f * g)(z)}{z}+\gamma(f * g)^{\prime}(z)\right] \\
& \prec(1-\rho) \cos \eta \cdot \frac{1+A z}{1+B z}+\rho \cos \eta+i \sin \eta, \\
& \left.\left(|\eta|<\frac{\pi}{2} ; 0 \leq \gamma \leq 1 ; 0 \leq \rho<1 ;-1 \leq B<A \leq 1 ; z \in \mathbb{U}\right)\right\}
\end{aligned}
$$

In this paper, we obtain the Fekete-Szegö inequalities for the functions in the class $S_{\gamma}^{b}(g(z) ; A, B)$. We also obtain an upper bound to the functional $H_{2}(2)$ for $f(z) \in S_{\gamma}^{b}(g(z) ; A, B)$. Earlier Janteng et al. [16], Mishra and Gochhayat [20], Mishra and Kund [21], Bansal [4] and many other authors have obtained sharp upper bounds of $H_{2}(2)$ for different classes of analytic functions.

2. Preliminaries. To prove our results, we need the following lemmas.

Lemma 1 ([26]). Let

$$
h(z)=1+\sum_{n=1}^{\infty} c_{n} z^{n} \prec 1+\sum_{n=1}^{\infty} C_{n} z^{n}=H(z) \quad(z \in \mathbb{U}) .
$$

If the function $H$ is univalent in $\mathbb{U}$ and $H(\mathbb{U})$ is a convex set, then

$$
\left|c_{n}\right| \leq\left|C_{1}\right| \text {. }
$$

Lemma 2 ([10]). Let a function $p \in \mathcal{P}$ be given by

$$
p(z)=1+c_{1} z+c_{2} z^{2}+\ldots \quad(z \in \mathbb{U}),
$$

then, we have

$$
\left|c_{n}\right| \leq 2 \quad(n \in \mathbb{N})
$$

The result is sharp.

Lemma 3 ([17, 18]). Let $p \in \mathcal{P}$ be given by the power series (2.3), then for any complex number $\nu$

$$
\left|c_{2}-\nu c_{1}^{2}\right| \leq 2 \max \{1 ;|2 \nu-1|\} .
$$

The result is sharp for the functions given by

$$
p(z)=\frac{1+z^{2}}{1-z^{2}} \text { and } p(z)=\frac{1+z}{1-z} \quad(z \in \mathbb{U}) .
$$

Lemma 4 ([15]). Let a function $p \in \mathcal{P}$ be given by the power series (2.3), then

$$
2 c_{2}=c_{1}^{2}+\varkappa\left(4-c_{1}^{2}\right)
$$

for some $\varkappa,|\varkappa| \leq 1$, and

$$
4 c_{3}=c_{1}^{3}+2\left(4-c_{1}^{2}\right) c_{1} \varkappa-c_{1}\left(4-c_{1}^{2}\right) \varkappa^{2}+2\left(4-c_{1}^{2}\right)\left(1-|\varkappa|^{2}\right) z,
$$

for some $z,|z| \leq 1$. 
3. Main results. We give the following result related to the coefficient of $f(z) \in S_{\gamma}^{b}(g(z) ; A, B)$.

Theorem 1. Let $f(z)$ given by (1.1) belong to the class $S_{\gamma}^{b}(g(z) ; A, B)$, $0 \leq \gamma \leq 1,-1 \leq B<A \leq 1$ and $b \in \mathbb{C}^{*}$, then

$$
\left|a_{k}\right| \leq \frac{(A-B)|b|}{[1+\gamma(k-1)] b_{k}} \quad(k \in \mathbb{N} \backslash\{1\}) .
$$

Proof. If $f(z)$ of the form (1.1) belongs to the class $S_{\gamma}^{b}(g(z) ; A, B)$, then

$$
1+\frac{1}{b}\left((1-\gamma) \frac{(f * g)(z)}{z}+\gamma(f * g)^{\prime}(z)-1\right) \prec \frac{1+A z}{1+B z}=h(z)
$$

$\left(b \in \mathbb{C}^{*} ; 0 \leq \gamma \leq 1 ;-1 \leq B<A \leq 1 ; z \in \mathbb{U}\right)$, where $h(z)$ is convex univalent in $\mathbb{U}$ and we have

$$
\begin{aligned}
& 1+\frac{1}{b}\left((1-\gamma) \frac{(f * g)(z)}{z}+\gamma(f * g)^{\prime}(z)-1\right) \\
& =1+\sum_{k=1}^{\infty} \frac{(1+k \gamma)}{b} b_{k+1} a_{k+1} z^{k} \prec 1+(A-B) z-B(A-B) z^{2}+\ldots
\end{aligned}
$$

$(z \in \mathbb{U})$. Now, by applying Lemma 1, we get the desired result.

Remark 1. Putting $g(z)=\frac{z}{1-z}$ in Theorem 1, we obtain the result obtained by Bansal [5, Theorem 2.1].

It is easy to derive a sufficient condition for $f(z)$ to be in the class $S_{\gamma}^{b}(m, \lambda, \ell ; A, B)$ using standard techniques (see [25]). Hence we state the following result without proof.

Theorem 2. Let $f(z) \in \mathcal{A}$, then a sufficient condition for $f(z)$ to be in the class $S_{\gamma}^{b}(g(z) ; A, B)$ is

$$
\sum_{k=2}^{\infty}[1+\gamma(k-1)] b_{k}\left|a_{k}\right| \leq \frac{(A-B)|b|}{1+B} .
$$

In the next two theorems, we obtain the result concerning Fekete-Szegö inequality and an upper bound for the Hankel determinant for the class $S_{\gamma}^{b}(g(z) ; A, B)$.

Remark 2. Putting $g(z)=\frac{z}{1-z}$ in Theorem 2, we obtain the result obtained by Bansal [5, Theorem 2.2].

Theorem 3. Let $f(z)$ given by (1.1) belong to the class $S_{\gamma}^{b}(g(z) ; A, B)$, $0 \leq \gamma \leq 1,-1 \leq B<A \leq 1$ and $b \in \mathbb{C}^{*}$, then

$$
\left|a_{3}-\mu a_{2}^{2}\right| \leq \frac{(A-B)|b|}{(1+2 \gamma) b_{3}} \cdot \max \left\{1,\left|B+\frac{\mu b b_{3}(A-B)(1+2 \gamma)}{(1+\gamma)^{2} b_{2}^{2}}\right|\right\} .
$$

This result is sharp. 
Proof. Let $f(z) \in S_{\gamma}^{b}(g(z) ; A, B)$, then there is a Schwarz function $w(z)$ in $U$ with $w(0)=0$ and $|w(z)|<1$ in $U$ and such that

$$
1+\frac{1}{b}\left((1-\gamma) \frac{(f * g)(z)}{z}+\gamma(f * g)^{\prime}(z)-1\right)=\Phi(w(z))
$$

$(z \in \mathbb{U})$, where

$$
\begin{aligned}
\Phi(z) & =\frac{1+A z}{1+B z}=1+(A-B) z-B(A-B) z^{2}+B^{2}(A-B) z^{3}-\ldots \\
& =1+B_{1} z+B_{2} z^{2}+B_{3} z^{3}+\ldots
\end{aligned}
$$

$(z \in \mathbb{U})$. If the function $p_{1}(z)$ is analytic and has positive real part in $U$ and $p_{1}(0)=1$, then

$$
p_{1}(z)=\frac{1+w(z)}{1-w(z)}=1+c_{1} z+c_{2} z^{2}+\ldots
$$

$(z \in \mathbb{U})$, since $w(z)$ is a Schwarz function. Define

$$
\begin{aligned}
h(z) & =1+\frac{1}{b}\left((1-\gamma) \frac{(f * g)(z)}{z}+\gamma(f * g)^{\prime}(z)-1\right) \\
& =1+d_{1} z+d_{2} z^{2}+\ldots
\end{aligned}
$$

$(z \in \mathbb{U})$. In view of the equations (3.5) and (3.7), we have

$$
p(z)=\Phi\left(\frac{p_{1}(z)-1}{p_{1}(z)+1}\right) .
$$

Since

$$
\frac{p_{1}(z)-1}{p_{1}(z)+1}=\frac{1}{2}\left[c_{1} z+\left(c_{2}-\frac{c_{1}^{2}}{2}\right) z^{2}+\left(c_{3}+\frac{c_{1}^{3}}{4}-c_{1} c_{2}\right) z^{3}+\ldots\right]
$$

we have

$$
\Phi\left(\frac{p_{1}(z)-1}{p_{1}(z)+1}\right)=1+\frac{1}{2} B_{1} c_{1} z+\left[\frac{1}{2} B_{1}\left(c_{2}-\frac{c_{1}^{2}}{2}\right)+\frac{1}{4} B_{2} c_{1}^{2}\right] z^{2}+\ldots
$$

and from this equation and (3.8), we obtain

$$
d_{1}=\frac{1}{2} B_{1} c_{1}, \quad d_{2}=\frac{1}{2} B_{1}\left(c_{2}-\frac{c_{1}^{2}}{2}\right)+\frac{1}{4} B_{2} c_{1}^{2}
$$

and

$$
d_{3}=\frac{B_{1}}{2}\left(c_{3}-c_{1} c_{2}+\frac{c_{1}^{3}}{4}\right)+\frac{B_{2} c_{1}}{2}\left(c_{2}-\frac{c_{1}^{2}}{2}\right)+\frac{B_{3} c_{1}^{3}}{8} .
$$

Then, from (3.6), we see that

$$
d_{1}=\frac{(1+\gamma) b_{2} a_{2}}{b} \text { and } d_{2}=\frac{(1+2 \gamma) b_{3} a_{3}}{b} .
$$


Now from (3.6), (3.8) and (3.13), we have

$$
a_{2}=\frac{(A-B) b c_{1}}{2(1+\gamma) b_{2}}, \quad a_{3}=\frac{b(A-B)}{4(1+2 \gamma) b_{3}}\left\{2 c_{2}-c_{1}^{2}(1+B)\right\}
$$

and

$$
a_{4}=\frac{b(A-B)}{8(1+3 \gamma) b_{4}}\left\{4 c_{3}-4 c_{1} c_{2}(1+B)+c_{1}^{3}(1+B)^{2}\right\}
$$

Therefore, we have

$$
a_{3}-\mu a_{2}^{2}=\frac{b(A-B)}{2(1+2 \gamma) b_{3}}\left\{c_{2}-\nu c_{1}^{2}\right\}
$$

where

$$
\nu=\frac{1}{2}\left[1+B+\frac{\mu b(A-B)(1+2 \gamma) b_{3}}{(1+\gamma)^{2} b_{2}^{2}}\right]
$$

Our result now follows by an application of Lemma 3. The result is sharp for the functions

$$
1+\frac{1}{b}\left((1-\gamma) \frac{(f * g)(z)}{z}+\gamma(f * g)^{\prime}(z)-1\right)=\Phi\left(z^{2}\right)
$$

and

$$
1+\frac{1}{b}\left((1-\gamma) \frac{(f * g)(z)}{z}+\gamma(f * g)^{\prime}(z)-1\right)=\Phi(z) .
$$

This completes the proof of Theorem 3 .

Remark 3. Putting $g(z)=\frac{z}{1-z}$ in Theorem 3, we obtain the result due to Bansal [5, Theorem 2.3].

Putting $g(z)=z+\sum_{k=2}^{\infty}\left[\frac{1+\ell+\lambda(k-1)}{1+\ell}\right]^{m} \Gamma_{k}\left(\alpha_{1}\right)\left(m \in \mathbb{N}_{0}, \ell \geq 0, \lambda \geq 0\right.$, $\left.q \leq s+1, q, s \in \mathbb{N}_{0}\right)$, where $\Gamma_{k}\left(\alpha_{1}\right)$ is given by (1.6) in Theorem 3, we obtain the following corollary.

Corollary 1. Let $f(z)$ given by (1.1) belong to the class $S_{\gamma}^{b}\left(\lambda, \ell, m, q, s, \alpha_{1}\right.$, $\left.\beta_{1} ; A, B\right), 0 \leq \gamma \leq 1,-1 \leq B<A \leq 1, m \in \mathbb{N}_{0}, \ell \geq 0, \lambda \geq 0, q \leq s+1$, $q, s \in \mathbb{N}_{0}$ and $b \in \mathbb{C}^{*}$, then

$$
\begin{aligned}
\left|a_{3}-\mu a_{2}^{2}\right| \leq & \frac{(A-B)(1+\ell)^{m}|b|}{(1+2 \gamma)(1+\ell+2 \lambda)^{m} \Gamma_{3}\left(\alpha_{1}\right)} \\
& \times \max \left\{1,\left|B+\frac{\mu b\left[\frac{1+\ell+2 \lambda}{1+\ell}\right]^{m} \Gamma_{3}\left(\alpha_{1}\right)(A-B)(1+2 \gamma)}{(1+\gamma)^{2}\left[\frac{1+\ell+\lambda}{1+\ell}\right]^{2 m} \Gamma_{2}^{2}\left(\alpha_{1}\right)}\right|\right\} .
\end{aligned}
$$

This result is sharp.

Putting $g(z)=z+\sum_{k=2}^{\infty}\left[\frac{1+\ell}{1+\ell+\lambda(k-1)}\right]^{m} z^{k}\left(m \in \mathbb{N}_{0} ; \ell \geq 0 ; \lambda \geq 0\right)$ in Theorem 3, we obtain the following corollary. 
Corollary 2. Let $f(z)$ given by (1.1) belong to the class $S_{\gamma}^{b}(\lambda, \ell, m ; A, B)$, $0 \leq \gamma \leq 1,-1 \leq B<A \leq 1, m \in \mathbb{N}_{0}, \ell \geq 0, \lambda \geq 0$ and $b \in \mathbb{C}^{*}$, then

$$
\begin{aligned}
\left|a_{3}-\mu a_{2}^{2}\right| \leq & \frac{(A-B)|b|}{(1+2 \gamma)}\left[\frac{1+\ell+2 \lambda}{1+\ell}\right]^{m} \\
& \times \max \left\{1,\left|B+\frac{\mu b\left[\frac{1+\ell}{1+\ell+2 \lambda}\right]^{m}(A-B)(1+2 \gamma)}{(1+\gamma)^{2}\left[\frac{1+\ell}{1+\ell+\lambda}\right]^{2 m}}\right|\right\} .
\end{aligned}
$$

This result is sharp.

Putting $b=(1-\rho) e^{-i \eta} \cos \eta\left(|\eta|<\frac{\pi}{2}, 0 \leq \rho<1\right)$ in Theorem 3, we obtain the following corollary.

Corollary 3. Let $f(z)$ given by (1.1) belong to the class $S^{\gamma}[\rho, \eta, A, B, g(z)]$, $0 \leq \gamma \leq 1,-1 \leq B<A \leq 1$ and $b \in \mathbb{C}^{*}$, then

$$
\begin{aligned}
\mid a_{3}- & \mu a_{2}^{2} \mid \leq \frac{(A-B)(1-\rho) \cos \eta}{(1+2 \gamma) b_{3}} \\
& \times \max \left\{1,\left|B+\frac{\mu b_{3}(A-B)(1+2 \gamma)(1-\rho) e^{-i \eta} \cos \eta}{(1+\gamma)^{2} b_{2}^{2}}\right|\right\} .
\end{aligned}
$$

This result is sharp.

Theorem 4. Let $f(z)$ given by (1.1) belong to the class $S_{\gamma}^{b}(g(z) ; A, B)$, $0 \leq \gamma \leq 1,-1 \leq B<A \leq 1$ and $b \in \mathbb{C}^{*}$, then

$$
\left|a_{2} a_{4}-a_{3}^{2}\right| \leq \frac{(A-B)^{2}|b|^{2}}{(1+2 \gamma)^{2} b_{3}^{2}}
$$

Proof. Using (3.14) and (3.15), we have

$$
\begin{aligned}
\left|a_{2} a_{4}-a_{3}^{2}\right|= & \frac{(A-B)^{2}|b|^{2}}{16(1+\gamma)(1+3 \gamma) b_{2} b_{4}} \mid 4 c_{1} c_{3}-4 c_{1}^{2} c_{2}(1+B)+c_{1}^{4}(1+B)^{2} \\
(3.24) \quad & -\frac{(1+\gamma)(1+3 \gamma) b_{2} b_{4}}{(1+2 \gamma)^{2} b_{3}^{2}}\left[4 c_{2}^{2}-4 c_{1}^{2} c_{2}(1+B)+c_{1}^{4}(1+B)^{2}\right] \mid \\
= & M \mid 4 c_{1} c_{3}-4 c_{1}^{2} c_{2}(1+B)+c_{1}^{4}(1+B)^{2} \\
& -N\left[4 c_{2}^{2}-4 c_{1}^{2} c_{2}(1+B)+c_{1}^{4}(1+B)^{2}\right] \mid
\end{aligned}
$$

where

$$
M=\frac{(A-B)^{2}|b|^{2}}{16(1+\gamma)(1+3 \gamma) b_{2} b_{4}} \text { and } N=\frac{(1+\gamma)(1+3 \gamma) b_{2} b_{4}}{(1+2 \gamma)^{2} b_{3}^{2}}
$$

The above equation (3.24) is equivalent to

$$
\left|a_{2} a_{4}-a_{3}^{2}\right|=M\left|4 c_{1} c_{3}+d_{2} c_{1}^{2} c_{2}+d_{3} c_{2}^{2}+d_{4} c_{1}^{4}\right|,
$$


where

(3.27) $d_{1}=4, \quad d_{2}=-4(1+B)(1-N), \quad d_{3}=-4 N, \quad d_{4}=(1-N)(1+B)^{2}$.

Since the functions $p(z)$ and $p\left(r e^{i \theta}\right)(\theta \in \mathbb{R})$ are members of the class $\mathcal{P}$ simultaneously, we assume without loss of generality that $c_{1}>0$. For convenience of notation, we take $c_{1}=c(c \in[0,2]$, see (2.4)). Also, substituting the values of $c_{2}$ and $c_{3}$, respectively, from (2.6) and (2.7) in (3.26), we have

$$
\begin{aligned}
\left|a_{2} a_{4}-a_{3}^{2}\right| & =\frac{M}{4} \mid c^{4}\left(d_{1}+2 d_{2}+d_{3}+4 d_{4}\right)+2 \varkappa c^{2}\left(4-c^{2}\right)\left(d_{1}+d_{2}+d_{3}\right) \\
& +\left(4-c^{2}\right) \varkappa^{2}\left(-d_{1} c^{2}+d_{3}\left(4-c^{2}\right)\right)+2 d_{1} c\left(4-c^{2}\right)\left(1-|\varkappa|^{2} z\right) \mid .
\end{aligned}
$$

An application of triangle inequality, replacement of $|\varkappa|$ by $\nu$ and substituting the values of $d_{1}, d_{2}, d_{3}$ and $d_{4}$ from (3.27), we have

$$
\begin{aligned}
\mid a_{2} a_{4}- & a_{3}^{2} \mid \leq \frac{M}{4}\left[4 c^{4}(1-N) B^{2}+8|B|(1-N) \nu c^{2}\left(4-c^{2}\right)\right. \\
& \left.+\left(4-c^{2}\right) \nu^{2}\left(4 c^{2}+4 N\left(4-c^{2}\right)\right)+8 c\left(4-c^{2}\right)\left(1-\nu^{2}\right)\right] \\
= & M\left[c^{4}(1-N) B^{2}+2 c\left(4-c^{2}\right)+2 \nu|B|(1-N) c^{2}\left(4-c^{2}\right)\right. \\
& \left.+\nu^{2}\left(4-c^{2}\right)\left(c^{2}(1-N)-2 c+4 N\right)\right] \\
= & F(c, \nu)
\end{aligned}
$$

Next, we assume that the upper bound for (3.28) occurs at an interior point of the rectangle $[0,2] \times[0,1]$. Differentiating $F(c, \nu)$ in $(3.28)$ partially with respect to $\nu$, we have

$$
\begin{aligned}
\frac{\partial F(c, \nu)}{\partial \nu}=M & {\left[2|B|(1-N) c^{2}\left(4-c^{2}\right)\right.} \\
& \left.+2 \nu\left(4-c^{2}\right)\left(c^{2}(1-N)-2 c+4 N\right)\right] .
\end{aligned}
$$

For $0<\nu<1$ and for any fixed $c$ with $0<c<2$, from (3.29), we observe that $\frac{\partial F}{\partial \nu}>0$. Therefore, $F(c, \nu)$ is an increasing function of $\nu$, which contradicts our assumption that the maximum value of $F(c, \nu)$ occurs at an interior point of the rectangle $[0,2] \times[0,1]$. Moreover, for fixed $c \in[0,2]$,

$$
\max F(c, \nu)=F(c, 1)=G(c) .
$$

Thus

$$
\begin{aligned}
G(c)= & M\left[c^{4}(1-N)\left(B^{2}-2|B|-1\right)\right. \\
& \left.+4 c^{2}(2|B|(1-N)+1-2 N)+16 N\right] .
\end{aligned}
$$

Next,

$$
\begin{aligned}
G^{\prime}(c) & =4 M c\left[c^{2}(1-N)\left(B^{2}-2|B|-1\right)+2(2|B|(1-N)+1-2 N]\right. \\
& =4 M c\left[c^{2}(1-N)\left(B^{2}-2|B|-1\right)+2\{(1-N)(2|B|+1)-N\}\right] .
\end{aligned}
$$


So $G^{\prime}(c)<0$ for $0<c<2$ and has a real critical point at $c=0$. Also $G(c)>G(2)$. Therefore, maximum of $G(c)$ occurs at $c=0$. Therefore, the upper bound of $F(c, \nu)$ corresponds to $\nu=1$ and $c=0$. Hence,

$$
\left|a_{2} a_{4}-a_{3}^{2}\right| \leq 16 M N=\frac{(A-B)^{2}|b|^{2}}{(1+2 \gamma)^{2} b_{3}^{2}} .
$$

This completes the proof of Theorem 4 .

Remark 4. (i) Putting $g(z)=\frac{z}{1-z}$ in Theorem 4, we obtain the result due to Bansal [5, Theorem 2.4];

(ii) Putting

$$
g(z)=z+\sum_{k=2}^{\infty} \frac{(\alpha)_{k-1}}{(\beta)_{k-1}} z^{k}
$$

$\left(\alpha \in \mathbb{C}, \beta \in \mathbb{C} \backslash \mathbb{Z}_{0}^{-}\right), b=(1-\rho) e^{-i \theta} \cos \theta\left(|\theta|<\frac{\pi}{2}, 0 \leq \rho<1\right), \gamma=0, A=1$ and $B=-1$ in Theorem 4 , we obtain the result due to Mishra and Kund [21, Theorem 3.1];

(iii) Putting

$$
g(z)=z+\sum_{k=2}^{\infty} \frac{(\lambda+1)_{k-1}}{(m)_{k-1}} k^{n} z^{k}
$$

$\left(m \in \mathbb{N} ; \lambda, n \in \mathbb{N}_{0}\right), b=(1-\rho) e^{-i \alpha} \cos \alpha\left(|\alpha|<\frac{\pi}{2} ; 0 \leq \sigma<1\right), \gamma=0$, $A=1$ and $B=-1$ in Theorem 4, we obtain the result due to Mohammed and Darus [22, Theorem 2.1];

(iv) Putting

$$
g(z)=z+\sum_{k=2}^{\infty}[1+(\alpha \mu k+\alpha-\mu)(k-1)]^{\sigma}(\rho)_{k-1} z^{k}
$$

$\left(0 \leq \mu \leq \alpha \leq 1, \rho, \sigma \in \mathbb{N}_{0}\right), b=\gamma=A=1$ and $B=-1$ in Theorem 4, we obtain the result due to Abubaker and Darus [1, Theorem 3.1].

Putting $g(z)=z+\sum_{k=2}^{\infty}\left[\frac{1+\ell+\lambda(k-1)}{1+\ell}\right]^{m} \Gamma_{k}\left(\alpha_{1}\right)\left(m \in \mathbb{N}_{0}, \ell \geq 0, \lambda \geq 0\right.$, $\left.q \leq s+1, q, s \in \mathbb{N}_{0}\right)$, where $\Gamma_{k}\left(\alpha_{1}\right)$ is given by (1.6) in Theorem 4, we obtain the following corollary.

Corollary 4. Let $f(z)$ given by (1.1) belong to the class $S_{\gamma}^{b}(\lambda, \ell, m, q, s$, $\left.\alpha_{1}, \beta_{1} ; A, B\right), 0 \leq \gamma \leq 1,-1 \leq B<A \leq 1, m \in \mathbb{N}_{0}, \ell \geq 0, \lambda \geq 0, q \leq s+1$, $q, s \in \mathbb{N}_{0}$ and $b \in \mathbb{C}^{*}$, then

$$
\left|a_{2} a_{4}-a_{3}^{2}\right| \leq \frac{(A-B)^{2}|b|^{2}}{(1+2 \gamma)^{2}\left[\frac{1+\ell+2 \lambda}{1+\ell}\right]^{2 m} \Gamma_{3}^{2}\left(\alpha_{1}\right)} .
$$

Putting $g(z)=z+\sum_{k=2}^{\infty}\left[\frac{1+\ell}{1+\ell+\lambda(k-1)}\right]^{m} z^{k}\left(m \in \mathbb{N}_{0} ; \ell \geq 0 ; \lambda \geq 0\right)$ in Theorem 4, we obtain the following corollary. 
Corollary 5. Let $f(z)$ given by (1.1) belong to the class $S_{\gamma}^{b}(\lambda, \ell, m ; A, B)$, $0 \leq \gamma \leq 1,-1 \leq B<A \leq 1, m \in \mathbb{N}_{0}, \ell \geq 0, \lambda \geq 0$ and $b \in \mathbb{C}^{*}$, then

$$
\left|a_{2} a_{4}-a_{3}^{2}\right| \leq \frac{(A-B)^{2}|b|^{2}}{(1+2 \gamma)^{2}\left[\frac{1+\ell}{1+\ell+2 \lambda}\right]^{2 m}}
$$

Putting $b=(1-\rho) e^{-i \eta} \cos \eta\left(|\eta|<\frac{\pi}{2}, 0 \leq \rho<1\right)$ in Theorem 4, we obtain the following corollary.

Corollary 6. Let $f(z)$ given by (1.1) belong to the class $S^{\gamma}[\rho, \eta, A, B, g(z)]$, $0 \leq \gamma \leq 1,-1 \leq B<A \leq 1$ and $b \in \mathbb{C}^{*}$, then

$$
\left|a_{2} a_{4}-a_{3}^{2}\right| \leq \frac{(A-B)^{2}(1-\rho)^{2} \cos ^{2} \eta}{(1+2 \gamma)^{2} b_{3}^{2}}
$$

\section{REFERENCES}

[1] Abubaker, A., Darus, M., Hankel determinant for a class of analytic functions involving a generalized linear differential operator, Internat. J. Pure Appl. Math. 69 (3) (2011), 429-435.

[2] Al-Oboudi, F. M., On univalent functions defined by a generalized Salagean operator, Internat. J. Math. Math. Sci. 27 (2004), 1429-1436.

[3] Aouf, M. K., Subordination properties for a certain class of analytic functions defined by the Salagean operator, Appl. Math. Lett. 22 (2009), 1581-1585.

[4] Bansal, D., Upper bound of second Hankel determinant for a new class of analytic functions, Appl. Math. Lett. 26 (1) (2013), 103-107.

[5] Bansal, D., Fekete-Szegö problem and upper bound of second Hankel determinant for a new class of analytic functions, Kyungpook Math. J. 54 (2014), 443-452.

[6] Bulboacă, T., Differential Subordinations and Superordinations, Recent Results, House of Scientific Book Publ., Cluj-Napoca, 2005.

[7] Cătaş, A., On certain classes of p-valent functions defined by multiplier transformations, in Proceedings of the International Symposium on Geometric Function Theory and Applications, (Istanbul, Turkey, August 2007), Istanbul, 2008, 241-250.

[8] Cho, N. E., Srivastava, H. M., Argument estimates of certain analytic functions defined by a class of multiplier transformations, Math. Comput. Modelling 37 (1-2) (2003), 39-49.

[9] Cho, N. E., Kim, T. H., Multiplier transformations and strongly close-to-convex functions, Bull. Korean Math. Soc. 40 (3) (2003), 399-410.

[10] Duren, P. L., Univalent Functions, Springer-Verlag, New York, 1983.

[11] Dziok, J., Srivastava, H. M., Classes of analytic functions associated with the generalized hypergeometric function, Appl. Math. Comput. 103 (1999), 1-13.

[12] Dziok, J., Srivastava, H. M., Certain subclasses of analytic functions associated with the generalized hypergeometric function, Integral Transforms Spec. Funct. 14 (2003), $7-18$.

[13] El-Ashwah, R. M., Aouf, M. K., Differential subordination and superordination for certain subclasses of p-valent functions, Math. Comput. Modelling 51 (2010), 349360.

[14] El-Ashwah, R. M., Aouf, M. K., Some properties of new integral operator, Acta Univ. Apulensis 24 (2010), 51-61.

[15] Grenander, U., Szegö, G., Toeplitz Forms and Their Application, Univ. of California Press, Berkeley, 1958. 
[16] Janteng, A., Halim, S. A., Darus, M., Hankel determinant for starlike and convex functions, Int. J. Math. Anal. (Ruse) 1 (13) (2007), 619-625.

[17] Keogh, F. R., Markes, E. P., A coefficient inequality for certain classes of analytic functions, Proc. Amer. Math. Soc. 20 (1969), 8-12.

[18] Libera, R. J., Złotkewicz, E. J., Coefficient bounds for the inverse of a function with derivative in $\mathcal{P}$, Proc. Amer. Math. Soc. 87 (2) (1983), 251-257.

[19] Miller, S. S., Mocanu, P. T., Differential Subordination: Theory and Applications, Marcel Dekker Inc., New York-Basel, 2000.

[20] Mishra, A. K., Gochhayat, P., Second Hankel determinant for a class of analytic functions defined by fractional derivative, Int. J. Math. Math. Sci. (2008), Art. ID 153280, 1-10.

[21] Mishra, A. K., Kund, S. N., Second Hankel determinant for a class of functions defined by the Carlson-Shaffer, Tamkang J. Math. 44 (1) (2013), 73-82.

[22] Mohammed, A., Darus, M., Second Hankel determinant for a class of analytic functions defined by a linear operator, Tamkang J. Math. 43 (3) (2012), 455-462.

[23] Noonan, J. W., Thomas, D. K., On the second Hankel determinant of areally mean p-valent functions, Trans. Amer. Math. Soc. 223 (2) (1976), 337-346.

[24] Prajapat, J. K., Subordination and superordination preserving properties for generalized multiplier transformation operator, Math. Comput. Modelling 55 (2012), $1456-1465$.

[25] Raina, R. K., Bansal, D., Some properties of a new class of analytic functions defined in tems of a Hadmard product, J. Inequal. Pure Appl. Math. 9 (2008), Art. 22, 1-9.

[26] Rogosinski, W., On the coefficients of subordinate functions, Proc. London Math. Soc. 48 (1943), 48-82.

[27] Sălăgean, G. S., Subclasses of univalent functions, in Complex analysis - fifth Romanian-Finnish seminar, Part 1 (Bucharest, 1981), Springer-Verlag, Berlin, 1983, $362-372$.

[28] Selvaraj, C., Karthikeyan, K. R., Differential subordinant and superordinations for certain subclasses of analytic functions, Far East J. Math. Sci. 29 (2) (2008), 419-430.

[29] Srivastava, H. M., Karlsson, P. W., Multiple Gaussian Hypergeometric Series, Ellis Horwood Limited, Chichester; Halsted Press, New York, 1985.
S. M. El-Deeb
Department of Mathematics
M. K. Aouf
Faculty of Science
Damietta University
Department of Mathematics
New Damietta 34517
Faculty of Science
Mansoura University
Egypt
Mansoura 35516
e-mail: shezaeldeeb@yahoo.com
Egypt
e-mail: mkaouf127@yahoo.com

Received April 21, 2015 